\title{
Optimum Power Allocation for Expected Achievable Rate Maximization with Outage Constraints in Cooperative Relay Networks
}

\author{
James C. F. Li and Subhrakanti Dey \\ ARC Special Research Centre for Ultra-Broadband Information Networks (CUBIN) \\ Department of Electrical and Electronic Engineering \\ University of Melbourne, Victoria 3010, Australia \\ Email: c.li, sdey@ee.unimelb.edu.au
}

\begin{abstract}
In this paper, we study an optimum power allocation problem for expected achievable rate maximization in a typical three node cooperative relay network with slow block fading channels. A long term average power and an outage probability threshold serve as the constraints on the problem. This is motivated by the fact that in many applications, a mixture of delay-sensitive and -insensitive data are transmitted and either maximizing expected achievable rate or minimizing the outage probability would probably not provide the desired solution. The problem considered in this paper (known as the "service outage based rate and power allocation" problem in literature) thus achieves a tradeoff between the two extremes of ergodic capacity and outage capacity. We show that the optimum power allocation scheme is a switched policy between two deterministic policies. Extensive numerical results are presented to demonstrate the benefits of cooperation as opposed to that of non-cooperation or direct transmission. We study the performance of the two simple but popular relaying schemes, namely, the amplify-and-forward and decode-andforward protocols. It is seen that these relaying protocols are extremely resilient against demanding outage probability constraints over a range of basic rate requirements and average power constraints.
\end{abstract}

\section{INTRODUCTION}

Recent years have seen a steady growth of research studies in the area of performance analysis of relay networks. Although relay networks were proposed in [1], and capacity bounds for such networks were extensively studied by Cover et. al. [2] in the 70's, it is due to the recent exciting applications of ad hoc and sensor networks, that relay networks have become popular again. Data transmission with relay(s) not only raises the achievable rate of information transmission, but also provides alternative routes when direct transmission is resource-consuming or totally infeasible. For wireless networks, the outage performance, defined as the probability of the instantaneous mutual information falling below a basic rate threshold, has been shown to improve dramatically due to the diversity gain offered by relay networks. This type of diversity has been named as cooperative diversity [3], which, as the term implies, is provided by the cooperation among various communication units. The authors of [3] proposed two simple schemes for relaying, namely the amplify and forward (AF) and decode and forward (DF) schemes amongst others. Various other schemes such as estimate and forward, compress and

This work was supported by the Australian Research Council. CUBIN is an affiliated program of National ICT Australia (NICTA) forward etc. have been also proposed in the literature. For a survey of these and other possible relaying protocols and their information theoretic capacity related results, see [4].

Analyzing the information theoretic capacity of fading wireless channels has been an important area of research over the past decade. Various notions of capacity for single-user block fading channels include ergodic capacity [5], delay-limited capacity [6] and capacity versus outage probability [7]. If the transmitter has the channel state information (CSI), then the transmit power can be controlled as a function of the channel to maximize the capacity. The paper [5] looked at the problem of maximizing the ergodic capacity subject to an average power constraint, and showed that the optimal power control law was waterfilling on the inverse of the channel gain (more power is allocated when the channel gain is high, than when the channel gain is low). The ergodic capacity can be achieved over a large number of channel realizations over time, and thus is of interest when data transmission takes place over a long period of time. In the case where delay needs to be small and data transmission is restricted over a small number of blocks of fading, the relevant notion of capacity is delay limited capacity or outage capacity. In [7], optimal power allocation for minimizing outage probability with short and long term power constraints was derived for a number of parallel fading channels. In the case of a typical three node (source-relay-destination) relay network, the equivalent notions of expected achievable rate and related optimal power allocation policies were studied in [8] (see also references therein), whereas [9] investigated the problem of optimal power allocation for outage probability minimization in relay networks.

The power allocation policies resulting from maximizing ergodic capacity (or expected achievable rate in the cooperative case) and from minimizing the probability of outage are very different and represent two ends of the spectrum. There are however, several variable-rate real-time applications for which different levels of quality of service, quantified in terms of the allocated rate for each of these levels, can be guaranteed for different applications. For example, in an application with simultaneous voice and data transmissions, as soon as a basic rate $r_{0}$ has been guaranteed for the voice service, any excess rate can be used to transmit data in a best effort fashion such that the long term average rate is maximized for the delay-insensitive data applications. Motivated by these practical considerations, in [10], [11], Luo et al. 
studied variable rate adaptive transmission schemes subject to a maximum outage probability constraint on a basic rate requirement in a slow fading $\mathrm{RF}$ environment. The notions of ergodic and outage capacity were judiciously combined in a service outage problem formulation, where the long term average rate was maximized subject to a maximum outage probability constraint on a basic rate requirement on top of the customary transmitter average power constraint.

In this paper, we extend the analysis of [11] to the case of a typical three node relay network for the AF and the DF schemes. We show that the optimum service outage constrained expected achievable rate can be attained with full CSI at the transmitter. The optimum power allocation to maximize the service outage constrained capacity is obtained as a switched scheme between two deterministic power allocation policies - the expected achievable rate maximizing power allocation policy and a basic rate power allocation policy. Numerical results compare this service outage capacity for the AF and the DF schemes with the direct transmission scheme (i.e, no co-operation from the relay node). These comparisons show that the $\mathrm{AF}$ and DF schemes are extremely robust (as compared to the direct transmission scheme) to the increasing basic rate requirements for a given outage probability constraint. They are also less power hungry as compared to the DT scheme for a given outage probability constraint. Overall, these results illustrate the benefits of cooperation in terms of the expected achievable rate under an outage and a long term average power constraint. Future work will investigate optimal power allocation schemes with partial or quantized feedback CSI at the transmitter for similar problems.

The remainder of the paper is organized as follows. Section II describes the network model and the underlying assumptions used through the paper. In Section III, we formulate the expected achievable rate problem as an optimization problem and provide some results derived from previous work. Afterwards, a thorough solution to the outage-constrained rate maximization is introduced in the form of a series of lemmas and a theorem in Section IV. Finally, the extensive numerical results and the concluding remarks are given in Section V and VI.

\section{Relay Network Model}

We consider a typical three node cooperative relay network where the source sends data to the destination with the help of a relay node, as shown in Figure 1, and the relay does not produce its own data. It is assumed that the channel gains for all the three links - Source-Destination (S-D), Source-Relay (S-R), and, Relay-Destination (R-D) - follow a typical block fading model. In this model, time is divided into blocks where each block spans a codeword of a large number of transmitted symbols. Within each such block, the channels are constant but they change from one block to another in an independent and identically distributed fashion. We assume that the links are statistically mutually independent but not necessarily identically distributed.

We consider the three common simple transmission schemes: Direct Transmission (DT), Amplify and Forward (AF) and Decode and Forward (DF). In the DT scheme, obviously the source transmits only directly to the destination, while in the $\mathrm{AF}$ and $\mathrm{DF}$ schemes, the source also uses

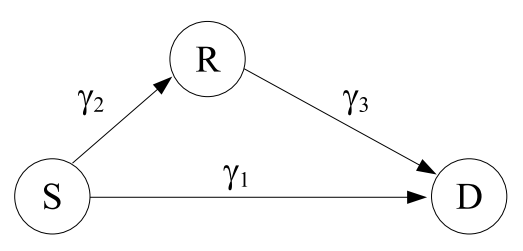

Fig. 1. A Relay Network with Random Fading Channels

the relay for data transmission. We assume a half-duplex time division transmission scheme where every transmission block (or fading block) is divided into two halves. During the first half, the source transmits to the relay and the destination. During the second half, the relay transmits to the destination and the source does not transmit. As assumed in [3], in the AF scheme, the relay only amplifies and forwards its received data to the destination. In the DF scheme, the relay first decodes and then forwards the decoded data to the destination. We also assume that there is no queue at the relay node. We use $\gamma_{1}, \gamma_{2}$, and $\gamma_{3}$ to denote the channel states of the S-D, S-R and R-D links respectively, and use the notation $\bar{\gamma}=\left(\gamma_{1}, \gamma_{2}, \gamma_{3}\right)$. It is assumed that the destination node has the exact knowledge of $\bar{\gamma}$ and feeds this information back to the source and the relay nodes through error free feedback channels with negligible delay. The source and relay transmit powers are denoted by $P_{s}(\bar{\gamma})$ and $P_{r}(\bar{\gamma})$ respectively.

\section{PROBLEM Formulation}

In this section, we present the main problem that we are interested in this paper. For a given average power limitation $P_{\mathrm{av}}$ and a required outage probability $\epsilon$, the service outage based allocation problem is

$$
\begin{gathered}
\max \mathbb{E}_{\bar{\gamma}}\left[R_{*}\left(P_{s}(\bar{\gamma}), P_{r}(\bar{\gamma}), \bar{\gamma}\right)\right] \\
\text { s.t. } \quad \mathbb{E}_{\bar{\gamma}}\left[P_{s}(\bar{\gamma})+P_{r}(\bar{\gamma})\right] \leq P_{\mathrm{av}} \\
\operatorname{Prob}\left\{R_{*}\left(P_{s}(\bar{\gamma}), P_{r}(\bar{\gamma}), \bar{\gamma}\right)<r_{0}\right\} \leq \epsilon \\
P_{s}(\bar{\gamma}), P_{r}(\bar{\gamma}) \geq 0
\end{gathered}
$$

where $r_{0}$ is the rate threshold for the outage, and the function $R_{*}\left(p_{s}, p_{r}, \bar{\gamma}\right)$ denotes the either the instantaneous mutual information (for the DT case) or the achievable rate (for the AF or the DF case), which are given below:

$$
\begin{aligned}
& R_{\mathrm{DT}}\left(p_{s}, p_{r}, \bar{\gamma}\right)=\log \left(1+\gamma_{1} p_{s}\right) \\
& R_{\mathrm{AF}}\left(p_{s}, p_{r}, \bar{\gamma}\right)=\frac{1}{2} \log \left(1+2 \gamma_{1} p_{s} \ldots\right. \\
&\left.+\frac{4 \gamma_{2} p_{s} \gamma_{3} p_{r}}{1+2 \gamma_{2} p_{s}+2 \gamma_{3} p_{r}}\right) \\
& R_{\mathrm{DF}}\left(p_{s}, p_{r}, \bar{\gamma}\right)=\frac{1}{2} \min \left\{\log \left(1+2 \gamma_{2} p_{s}\right), \ldots\right. \\
&\left.\log \left(1+2 \gamma_{1} p_{s}+2 \gamma_{3} p_{r}\right)\right\} .
\end{aligned}
$$

The maximum expected achievable rate in Problem (1) is termed as the service outage based achievable rate or more loosely, the service outage capacity. The expression above for $R_{\mathrm{AF}}\left(p_{s}, p_{r}, \bar{\gamma}\right)$ is not jointly concave in $p_{s}, p_{r}$. Hence we will use the popular high SNR approximation $1+2 \gamma_{2} P_{s}+2 \gamma_{3} P_{r} \approx 2 \gamma_{2} P_{s}+2 \gamma_{3} P_{r}$ resulting in the following approximate expression for $R_{\mathrm{AF}}\left(p_{s}, p_{r}, \bar{\gamma}\right) \approx$ $\frac{1}{2} \log \left(1+2 \gamma_{1} p_{s}+\frac{2 \gamma_{2} p_{s} \gamma_{3} p_{r}}{\gamma_{2} p_{s}+\gamma_{3} p_{r}}\right)$, which is jointly concave in $p_{s}, p_{r}$. We will use this approximation for the achievable 
rate in the AF case for the rest of the paper. Note however that $R_{\mathrm{DF}}\left(p_{s}, p_{r}, \bar{\gamma}\right)$ is jointly concave in $p_{s}, p_{r}$. We will use the notation $[x]^{+}$to denote $\max (x, 0)$ and the notation $\langle v\rangle$ to denote the sum of the elements of the vector $v$. Note also that the problem (1) was solved in [10] for the direct transmission case for a single fading channel and then later extended to [11] for parallel fading channels. In this paper, we adopt the techniques of [11] in order to solve (1) for the relay channel using the $\mathrm{AF}$ or DF relaying protocols.

Before we can provide the solution to (1), it is instructive to note the solutions to the expected achievable rate maximization problem stated as follows:

$$
\begin{array}{ll}
\max & \mathbb{E}_{\bar{\gamma}}\left[R_{*}\left(P_{s}(\bar{\gamma}), P_{r}(\bar{\gamma}), \bar{\gamma}\right)\right] \\
\text { s.t. } & \mathbb{E}_{\bar{\gamma}}\left[P_{s}(\bar{\gamma})+P_{r}(\bar{\gamma})\right] \leq P_{\mathrm{av}} \\
& P_{s}(\bar{\gamma}), P_{r}(\bar{\gamma}) \geq 0
\end{array}
$$

and the basic rate allocation problem stated as follows:

$$
\begin{array}{ll} 
& \min P_{s}(\bar{\gamma})+P_{r}(\bar{\gamma}) \\
\text { s.t. } \quad & R_{*}\left(P_{s}(\bar{\gamma}), P_{r}(\bar{\gamma}), \bar{\gamma}\right) \geq r_{0}, \\
& P_{s}(\bar{\gamma}), P_{r}(\bar{\gamma}) \geq 0 .
\end{array}
$$

We denote the solutions to Problem (3) in the AF case as $P_{\mathrm{AF}}^{\mathrm{erg}}\left(\bar{\gamma}, \lambda^{*}\right)=\left(P_{s: A F}^{\mathrm{erg}}, P_{r: A F}^{\mathrm{erg}}\right)$ and in the DF case as $P_{\mathrm{DF}}^{\mathrm{erg}}\left(\bar{\gamma}, \lambda^{*}\right)=\left(P_{\mathrm{s}: \mathrm{DF}}^{\mathrm{erg}}, P_{\mathrm{r}: \mathrm{DF}}^{\mathrm{erg}}\right)$ (due to its similarity with the ergodic capacity maximization problem for fading channels) and the solutions to Problem (4) in the AF case as $P_{\mathrm{AF}}^{r_{0}}(\bar{\gamma})=$ $\left(P_{\mathrm{s}: \mathrm{AF}}^{r_{0}}, P_{\mathrm{r}: \mathrm{AF}}^{r_{0}}\right)$ and in the DF case as $P_{\mathrm{DF}}^{r_{0}}(\bar{\gamma})=\left(P_{\mathrm{s}: \mathrm{DF}}^{r_{0}}, P_{\mathrm{r}: \mathrm{DF}}^{r_{0}}\right)$. The solutions to these problems have appeared in various earlier work in different forms. We refer to [12] and [8] for further details. For the AF case, these solutions are given by

$$
\begin{aligned}
& P_{\mathrm{AF}}^{\mathrm{erg}}\left(\bar{\gamma}, \lambda^{*}\right) \\
& = \begin{cases}\left(\left[\frac{1}{2 \lambda^{*}}-\frac{1}{2 \gamma_{1}}\right]^{+}, 0\right) & \gamma_{1} \geq \gamma_{3} \\
\left(\left[\frac{1}{2 \lambda^{*}}-\frac{1}{\frac{2 u}{1+u}\left(\gamma_{1}+\frac{\gamma_{2} \gamma_{3}}{\gamma_{2} u+\gamma_{3}}\right)}\right]^{+} \frac{u}{1+u}, \ldots\right. & \\
\left.\quad\left[\frac{1}{2 \lambda^{*}}-\frac{2 u}{\frac{2 u}{1+u}\left(\gamma_{1}+\frac{1}{\gamma_{2} u+\gamma_{3}}\right)}\right]^{+} \frac{1}{1+u}\right) & \gamma_{1}<\gamma_{3}\end{cases}
\end{aligned}
$$

where the ratio of $P_{\mathrm{s}: \mathrm{AF}}^{\mathrm{erg}} / P_{\mathrm{r:AF}}^{\mathrm{erg}}, u=\frac{\gamma_{3}\left(\gamma_{1}+\sqrt{\gamma_{1} \gamma_{3}-\gamma_{1} \gamma_{2}+\gamma_{2} \gamma_{3}}\right)}{\left(\gamma_{3}-\gamma_{1}\right) \gamma_{2}}$, and $\lambda^{*}$ is the Lagrange multiplier corresponding the average power constraint.

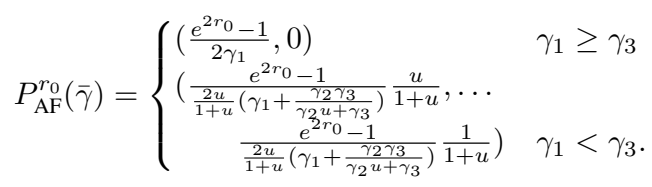

Similarly, for the DF case, these solutions are given by

$$
\begin{aligned}
& P_{\mathrm{DF}}^{\mathrm{erg}}\left(\bar{\gamma}, \lambda^{*}\right) \\
& = \begin{cases}\left(\left[\frac{1}{2 \lambda^{*}}-\frac{1}{2 \gamma_{2}}\right]^{+}, 0\right) & \gamma_{1}>\gamma_{2} \\
\left(\left[\frac{1}{2 \lambda^{*}}-\frac{1}{2 \gamma_{1}}\right]^{+}, 0\right) & \gamma_{1} \leq \gamma_{2} \quad \gamma_{1}>\gamma_{3} \\
\left(\left[\frac{1}{2 \lambda^{*}}-\frac{\gamma_{2}+\gamma_{3}-\gamma_{1}}{2 \gamma_{2} \gamma_{3}}\right]^{+} \frac{\gamma_{3}}{\gamma_{2}+\gamma_{3}-\gamma_{1}}, \ldots\right. & \\
\left.\quad\left[\frac{1}{2 \lambda^{*}}-\frac{\gamma_{2}+\gamma_{3}-\gamma_{1}}{2 \gamma_{2} \gamma_{3}}\right]^{+} \frac{\gamma_{2}-\gamma_{1}}{\gamma_{2}+\gamma_{3}-\gamma_{1}}\right) & \gamma_{1} \leq \min \left\{\gamma_{2}, \gamma_{3}\right\}\end{cases}
\end{aligned}
$$

and

$$
P_{\mathrm{DF}}^{r_{0}}(\bar{\gamma})= \begin{cases}\left(\frac{e^{2 r_{0}}-1}{2 \gamma_{2}}, 0\right) & \gamma_{1}>\gamma_{2} \\ \left(\frac{e^{2 r_{0}}-1}{2 \gamma_{1}}, 0\right) & \gamma_{1} \leq \gamma_{2} \quad \gamma_{1}>\gamma_{3} \\ \left(\frac{e^{2 r_{0}}-1}{2 \gamma_{2}}, \frac{\left(e^{2 r_{0}}-1\right)\left(\gamma_{2}-\gamma_{1}\right)}{2 \gamma_{2} \gamma_{3}}\right) & \gamma_{1} \leq \min \left\{\gamma_{2}, \gamma_{3}\right\}\end{cases}
$$

The basic rate power allocation solutions are intimately related to the outage probability minimizing solution, more details on which can be found in [9], [13]. It should be obvious that in order for Problem (1) to be feasible for a given $r_{0}, \epsilon$, there is a minimum average power necessary denoted by $P_{\min }\left(r_{0}, \epsilon\right)$. We assume for the rest of the paper that $P_{\mathrm{av}}>P_{\min }\left(r_{0}, \epsilon\right)$. When $P_{\mathrm{av}}$ is exactly equal to $P_{\min }\left(r_{0}, \epsilon\right)$, the optimal power allocation is essentially the basic rate allocation when the instantaneous sum of source and relay power is less than a certain threshold (determined by $P_{\min }\left(r_{0}, \epsilon\right)$ ), and zero above this threshold, with a suitable randomization at the boundary. This randomization is not necessary for continuous fading distributions, see [11] for more details.

\section{Optimum Power Allocation Scheme}

In this section we show that the optimum power allocation scheme that solves Problem (1) is a randomized solution between two deterministic schemes involving the expected achievable rate maximizing power allocation and the basic rate power allocation. In what follows, we will present the derivation of the optimum power allocation scheme for the AF case. The exact same technique can be applied to obtain the results for the DF case.

Before we can proceed, we define a Bernoulli random variable $X_{w}(\bar{\gamma})$ for a given $\bar{\gamma}$ which takes value 1 with probability $w(\bar{\gamma})$ and value 0 with probability $1-w(\bar{\gamma})$. By using the concavity of $R_{\mathrm{AF}}\left(P_{\mathrm{s}: \mathrm{AF}}(\bar{\gamma}), P_{\mathrm{r}: \mathrm{AF}}(\bar{\gamma}), \bar{\gamma}\right)$ (the high SNR approximation), the following result can be proved (see [11] for a similar proof for the direct transmission case).

Lemma 4.1: There exists an optimal solution for problem (1) of the following form:

$$
\begin{aligned}
P_{\mathrm{AF}}^{*}(\bar{\gamma})= & X_{w}(\bar{\gamma}) P_{\mathrm{AF}}^{\text {in:* }}(\bar{\gamma})+\left(1-X_{w}(\bar{\gamma})\right) P_{\mathrm{AF}}^{\text {out: } *}(\bar{\gamma}) \\
& P_{\mathrm{AF}}^{*}(\bar{\gamma}) \triangleq\left(P_{s: \mathrm{AF}}^{*}(\bar{\gamma}), P_{r: \mathrm{AF}}^{*}(\bar{\gamma})\right) \\
& P_{\mathrm{AF}}^{\text {in: } *}(\bar{\gamma}) \triangleq\left(P_{s: \mathrm{AF}}^{\text {in: } *}(\bar{\gamma}), P_{r: \mathrm{AF}}^{\text {in:* }}(\bar{\gamma})\right) \\
& P_{\mathrm{AF}}^{\text {out: } *}(\bar{\gamma}) \triangleq\left(P_{s: \mathrm{AF}}^{\text {out: } *}(\bar{\gamma}), P_{r: \mathrm{AF}}^{\text {out: } *}(\bar{\gamma})\right)
\end{aligned}
$$

where $R_{\mathrm{AF}}\left(P_{s: \mathrm{AF}}^{\text {in }}(\bar{\gamma}), P_{r: \mathrm{AF}}^{\text {in }}(\bar{\gamma}), \bar{\gamma}\right) \geq r_{0}$ for all $\bar{\gamma}$, $\mathbb{E}_{\bar{\gamma}}[w(\bar{\gamma})] \geq 1-\epsilon$, which is called 'in' region, and, the power allocated in this region is denoted as $P_{\mathrm{AF}}^{\text {in:* }}$. Similarly, $P_{\mathrm{AF}}^{\text {out:* }}$ symbolizes the power allocated in the 'outage' region. In addition, $\mathbb{E}_{\bar{\gamma}}\left[\left\langle P_{\mathrm{AF}}^{*}(\bar{\gamma})\right\rangle\right]=\mathbb{E}_{\bar{\gamma}}\left[P_{s: \mathrm{AF}}^{*}(\bar{\gamma})+P_{r: \mathrm{AF}}^{*}(\bar{\gamma})\right]=P_{\mathrm{av}}$ (where recall that $\langle\bullet\rangle$ is defined as the sum of the transmit powers at the source and the relay).

Note that here $P_{\mathrm{AF}}^{\mathrm{in}: *}(\bar{\gamma})$ and $P_{\mathrm{AF}}^{\text {out: } *}(\bar{\gamma})$ are deterministic power allocation schemes to be decided. The above Lemma allows the original optimization problem to be rewritten as the following optimization problem over $P_{\mathrm{AF}}^{\mathrm{in}}(\bar{\gamma}), P_{\mathrm{AF}}^{\text {out }}(\bar{\gamma})$ and $w(\bar{\gamma})$ :

$$
\begin{gathered}
\max _{P_{\mathrm{AF}}^{\text {in }}, P_{\mathrm{AF}}^{\text {out }}, w} \mathbb{E}_{\bar{\gamma}}\left[w(\bar{\gamma}) R_{\mathrm{AF}}\left(P_{s: \mathrm{AF}}^{\text {in }}(\bar{\gamma}), P_{r: \mathrm{AF}}^{\text {in }}(\bar{\gamma}), \bar{\gamma}\right) \ldots\right. \\
\left.+(1-w(\bar{\gamma})) R_{\mathrm{AF}}\left(P_{s: \mathrm{AF}}^{\text {out }}(\bar{\gamma}), P_{r: \mathrm{AF}}^{\text {out }}(\bar{\gamma}), \bar{\gamma}\right)\right] \\
\text { s.t. } \quad \mathbb{E}_{\bar{\gamma}}\left[w(\bar{\gamma})\left\langle P_{\mathrm{AF}}^{\text {in }}(\bar{\gamma})\right\rangle+(1-w(\bar{\gamma}))\left\langle P_{\mathrm{AF}}^{\text {out }}(\bar{\gamma})\right\rangle\right] \leq P_{\mathrm{av}} \\
\mathbb{E}_{\bar{\gamma}}[w(\bar{\gamma})] \geq 1-\epsilon \\
R_{\mathrm{AF}}\left(P_{s: \mathrm{AF}}^{\text {in }}(\bar{\gamma}), P_{r: \mathrm{AF}}^{\text {in }}(\bar{\gamma}), \bar{\gamma}\right) \geq r_{0} \\
P_{s: \mathrm{AF}}^{\text {in }}(\bar{\gamma}), P_{r: \mathrm{AF}}^{\text {in }}(\bar{\gamma}), P_{s: \mathrm{AF}}^{\text {out }}(\bar{\gamma}), P_{r: \mathrm{AF}}^{\text {out }}(\bar{\gamma}) \geq 0 \\
0 \leq w(\bar{\gamma}) \leq 1
\end{gathered}
$$


Let us assume $\left(P_{\mathrm{AF}}^{\text {in: } *}(\bar{\gamma}), P_{\mathrm{AF}}^{\text {out } *}(\bar{\gamma}), w^{*}(\bar{\gamma})\right)$ optimize Problem (10) and $\left(\lambda^{*}, \beta^{*}, u^{*}(\bar{\gamma})\right) \geq 0$ are the corresponding Lagrange multipliers. If we eliminate the items which do not contain primary variables, the Lagrange function can be written as

$$
\begin{aligned}
L & \left(P_{\mathrm{AF}}^{\text {in }}(\bar{\gamma}), P_{\mathrm{AF}}^{\text {out }}(\bar{\gamma}), w(\bar{\gamma}), \lambda, \beta, u(\bar{\gamma}), \bar{\gamma}\right) \\
=w & (\bar{\gamma}) R_{\mathrm{AF}}\left(P_{s: \mathrm{AF}}^{\text {in }}(\bar{\gamma}), P_{r: \mathrm{AF}}^{\text {in }}(\bar{\gamma}), \bar{\gamma}\right) \\
& +(1-w(\bar{\gamma})) R_{\mathrm{AF}}\left(P_{s: \mathrm{AF}}^{\text {out }}(\bar{\gamma}), P_{r: \mathrm{AF}}^{\text {out }}(\bar{\gamma}), \bar{\gamma}\right) \\
& -\lambda\left(w(\bar{\gamma})\left(P_{s: \mathrm{AF}}^{\text {in }}(\bar{\gamma})+P_{r: \mathrm{AF}}^{\text {in }} \bar{\gamma}\right)\right) \\
& \left.+(1-w(\bar{\gamma}))\left(P_{s: \mathrm{AF}}^{\text {out }}(\bar{\gamma})+P_{r: \mathrm{AF}}^{\text {out }}(\bar{\gamma})\right)\right) \\
& +\beta w(\bar{\gamma})+u(\bar{\gamma}) R_{\mathrm{AF}}\left(P_{s: \mathrm{AF}}^{\text {in }}(\bar{\gamma}), P_{r: \mathrm{AF}}^{\text {in }}(\bar{\gamma}), \bar{\gamma}\right) .
\end{aligned}
$$

Therefore, from the KKT necessary conditions, the optimal solution $\left(P_{\mathrm{AF}}^{\text {in: } *}(\bar{\gamma}), P_{\mathrm{AF}}^{\text {out:* }}(\bar{\gamma}), w^{*}(\bar{\gamma}), \lambda^{*}, \beta^{*}, u^{*}(\bar{\gamma})\right)$ must satisfy

$$
\begin{aligned}
& \frac{\partial L(\ldots)}{\partial P_{s: A F}^{\text {in:* }}(\bar{\gamma})} \begin{cases}=0 & P_{s: A F}^{\text {in } * *}(\bar{\gamma})>0 \\
\leq 0 & P_{s: A F}^{\text {in: }}(\bar{\gamma})=0\end{cases} \\
& \frac{\partial L(\ldots)}{\partial P_{r: \mathrm{AF}}^{\mathrm{in} * *}(\bar{\gamma})} \begin{cases}=0 & P_{r: \mathrm{AF}}^{\mathrm{in} *}(\bar{\gamma})>0 \\
\leq 0 & P_{r: \mathrm{AF}}^{\mathrm{in} * *}(\bar{\gamma})=0\end{cases} \\
& \frac{\partial L(\ldots)}{\partial P_{s: \mathrm{AF}}^{\text {out: }}(\bar{\gamma})} \begin{cases}=0 & P_{s: \mathrm{AF}}^{\text {out } *}(\bar{\gamma})>0 \\
\leq 0 & P_{s: \mathrm{AF}}^{\text {out: }}(\bar{\gamma})=0\end{cases} \\
& \frac{\partial L(\ldots)}{\partial P_{r: \mathrm{AF}}^{\text {out:*}}(\bar{\gamma})} \begin{cases}=0 & P_{r: \mathrm{AF}}^{\text {out } *}(\bar{\gamma})>0 \\
\leq 0 & P_{r: \mathrm{AF}}^{\text {out: }}(\bar{\gamma})=0\end{cases} \\
& \frac{\partial L(\ldots)}{\partial w^{*}(\bar{\gamma})} \begin{cases}=0 & 0<w^{*}(\bar{\gamma})<1 \\
\leq 0 & w^{*}(\bar{\gamma})=0 \\
\geq 0 & w^{*}(\bar{\gamma})=1\end{cases} \\
& \left.u^{*}(\bar{\gamma})\right)\left[R_{\mathrm{AF}}\left(P_{s: \mathrm{AF}}^{\mathrm{in} * *}(\bar{\gamma}), P_{r: \mathrm{AF}}^{\mathrm{in}: *}(\bar{\gamma}), \gamma\right)-r_{0}\right]=0 \\
& \beta^{*}\left[\mathbb{E}_{\bar{\gamma}}\left[w^{*}(\bar{\gamma})\right]-(1-\epsilon)\right]=0 \\
& \mathbb{E}_{\gamma}\left[w^{*}(\bar{\gamma})\left\langle P_{\mathrm{AF}}^{\text {in: } *}(\bar{\gamma})\right\rangle+\left(1-w^{*}(\bar{\gamma})\right)\left\langle P_{\mathrm{AF}}^{\text {out: } *}(\bar{\gamma})\right\rangle\right]=P_{\mathrm{av}} .
\end{aligned}
$$

where we have used the shorthand notation $L(\ldots)$ for the Lagrangian in (11). By defining variables

$$
\begin{aligned}
& Q_{s: \mathrm{AF}}^{\text {in }}(\bar{\gamma})=w(\bar{\gamma}) P_{s: \mathrm{AF}}^{\text {in }}(\bar{\gamma}) \\
& Q_{r: \mathrm{AF}}^{\text {in }}(\bar{\gamma})=w(\gamma) P_{r: \mathrm{AF}}^{\text {in }}(\bar{\gamma}) \\
& Q_{s: \mathrm{AF}}^{\text {out }}(\bar{\gamma})=(1-w(\bar{\gamma})) P_{s: \mathrm{AF}}^{\text {out }}(\bar{\gamma}) \\
& Q_{r: \mathrm{AF}}^{\text {out }}(\bar{\gamma})=(1-w(\bar{\gamma})) P_{r: \mathrm{AF}}^{\text {out }}(\bar{\gamma})
\end{aligned}
$$

we can easily transform the optimization problem (10) as follows:

$$
\begin{gathered}
\max _{Q_{\mathrm{AF}}^{\text {in }}, Q_{\mathrm{AF}}^{\text {out }}, w} \mathbb{E}_{\bar{\gamma}}\left[w(\bar{\gamma}) R_{\mathrm{AF}}\left(\frac{Q_{s: \mathrm{AF}}^{\text {in }}(\bar{\gamma})}{w(\bar{\gamma})}, \frac{Q_{r: \mathrm{AF}}^{\text {in }}(\bar{\gamma})}{w(\bar{\gamma})}, \bar{\gamma}\right) \ldots\right. \\
\left.+(1-w(\bar{\gamma})) R_{\mathrm{AF}}\left(\frac{Q_{s: \mathrm{AF}}^{\text {out }}(\bar{\gamma})}{(1-w(\bar{\gamma}))}, \frac{Q_{r: \mathrm{AF}}^{\text {out }}(\bar{\gamma})}{(1-w(\bar{\gamma}))}, \bar{\gamma}\right)\right] \\
\text { s.t. } \quad \mathbb{E}_{\bar{\gamma}}\left[Q_{s: \mathrm{AF}}^{\text {in }}(\bar{\gamma})+Q_{r: \mathrm{AF}}^{\text {in }}(\bar{\gamma}) \ldots\right. \\
\left.+Q_{s: \mathrm{AF}}^{\text {out }}(\bar{\gamma})+Q_{r: \mathrm{AF}}^{\text {out }}(\bar{\gamma})\right] \leq P_{\mathrm{av}} \\
\mathbb{E}_{\gamma}[w(\bar{\gamma})] \geq 1-\epsilon \\
\begin{array}{c}
w(\bar{\gamma}) R_{\mathrm{AF}}\left(\frac{Q_{s: \mathrm{AF}}^{\text {in }}(\bar{\gamma})}{w(\bar{\gamma})}, \frac{Q_{r: \mathrm{AF}}^{\text {in }}(\bar{\gamma})}{w(\bar{\gamma})}, \bar{\gamma}\right)-w(\bar{\gamma}) r_{0} \geq 0 \\
Q_{s: \mathrm{AF}}^{\text {in }}(\bar{\gamma}), Q_{r: \mathrm{AF}}^{\text {in }}(\bar{\gamma}), Q_{s: \mathrm{AF}}^{\text {out }}(\bar{\gamma}), Q_{r: \mathrm{AF}}^{\text {out }}(\bar{\gamma}) \geq 0 \\
0 \leq w(\bar{\gamma}) \leq 1
\end{array}
\end{gathered}
$$

It is clear that the constraints (14), (15), (17), and (18) are linear constraints. We also know from [14] that the following property holds:

Proposition 1: If $f: \mathbb{R}^{n} \rightarrow \mathbb{R}$, then the perspective of $f$ is the function $g: \mathbb{R}^{n+1} \rightarrow \mathbb{R}$ defined by $g(t, x)=t f\left(\frac{x}{t}\right)$ with domain

$$
\operatorname{dom}\{g\}=\left\{(t, x): \frac{x}{t} \in \operatorname{dom}\{f\}, t>0\right\} .
$$

The perspective operation preserves convexity: if $f$ is a convex function, then so is its perspective function $g$. Similarly, if $f$ is concave, then so is $g$.

By using the above fact and the fact that the achievable rate function for $\mathrm{AF}$ (and DF) are concave functions, one can show that the objective function and constraint (16) are concave as well and therefore Problem (13) is a convex optimization problem. Hence the KKT conditions for (13) are necessary and sufficient. In addition, variables $P_{\mathrm{AF}}^{\mathrm{in}}$ and $P_{\mathrm{AF}}^{\text {out }}$ are determined by one-to-one functions implying that the KKT conditions are also necessary and sufficient for (10).

Lemma 4.2: The optimal values of the transmit powers for the source and the relay are

$$
\begin{aligned}
P_{\mathrm{AF}}^{\text {in:* }}(\bar{\gamma}) & = \begin{cases}P_{\mathrm{AF}}^{\mathrm{erg}}\left(\bar{\gamma}, \lambda^{*}\right) & R_{\mathrm{AF}}\left(P_{\mathrm{AF}}^{\mathrm{in} * *}(\bar{\gamma}), \bar{\gamma}\right) \geq r_{0} \\
P_{\mathrm{AF}}^{r_{0}}(\bar{\gamma}) & \text { otherwise }\end{cases} \\
P_{\mathrm{AF}}^{\text {out }}(\bar{\gamma}) & =P_{\mathrm{AF}}^{\mathrm{erg}}\left(\bar{\gamma}, \lambda^{*}\right)
\end{aligned}
$$

where $P_{\mathrm{AF}}^{\mathrm{erg}}\left(\bar{\gamma}, \lambda^{*}\right), P_{\mathrm{AF}}^{r_{0}}(\bar{\gamma})$ are given by (5) and (6).

Similar results can be obtained for the DF case. All that remains now is to obtain the optimal solution for $w^{*}(\bar{\gamma})$.

It is easy to notice that $P_{\mathrm{AF}}^{\mathrm{in}: *}(\bar{\gamma})$ can be expressed as

$$
P_{\mathrm{AF}}^{\mathrm{in}: *}(\bar{\gamma})=P_{\mathrm{AF}}^{\mathrm{erg}}\left(\bar{\gamma}, \lambda^{*}\right)+\left[P_{\mathrm{AF}}^{r_{0}}(\bar{\gamma})-P_{\mathrm{AF}}^{\mathrm{erg}}\left(\bar{\gamma}, \lambda^{*}\right)\right]^{+}
$$

and the rate achieved by $P_{\mathrm{AF}}^{\mathrm{in} * *}(\bar{\gamma})$ can be represented by

$$
R_{\mathrm{AF}}\left(P_{\mathrm{AF}}^{\mathrm{in}: *}(\bar{\gamma}), \bar{\gamma}\right)=R_{\mathrm{AF}}\left(P_{\mathrm{AF}}^{\mathrm{erg}}\left(\bar{\gamma}, \lambda^{*}\right), \bar{\gamma}\right)+R_{\text {supp }}\left(\bar{\gamma}, \lambda^{*}\right)
$$

where $R_{\text {supp }}\left(\bar{\gamma}, \lambda^{*}\right)=\left[r_{0}-R_{\mathrm{AF}}\left(P_{\mathrm{AF}}^{\mathrm{erg}}\left(\bar{\gamma}, \lambda^{*}\right)\right]^{+}\right.$is called the supplemental rate and $P_{\text {supp }}\left(\bar{\gamma}, \lambda^{*}\right)=\left[P_{\mathrm{AF}}^{r_{0}}(\bar{\gamma})-\right.$ $\left.P_{\mathrm{AF}}^{\mathrm{erg}}\left(\bar{\gamma}, \lambda^{*}\right)\right]^{+}$is called the supplemental power allocation. Essentially, the supplemental power provides the additional power needed to meet the basic rate $r_{0}$ when the expected achievable rate maximizing power allocation cannot meet this rate. Similarly, the supplemental rate is the additional rate needed to meet the basic rate on top of that achieved by the expected achievable rate maximizing power allocation [11]. Thus, the optimal power allocation can be expressed as

$$
P_{\mathrm{AF}}^{*}(\bar{\gamma})=P_{\mathrm{AF}}^{\mathrm{erg}}\left(\bar{\gamma}, \lambda^{*}\right)+X_{w^{*}}(\bar{\gamma}) P_{\text {supp }}\left(\bar{\gamma}, \lambda^{*}\right)
$$

In order to determine $w^{*}(\bar{\gamma})$, one can now rewrite the Lagrangian (11) as $w^{*}(\bar{\gamma})\left[\beta^{*}-g_{\mathrm{AF}}\left(\bar{\gamma}, \lambda^{*}\right)\right]+$ $R_{\mathrm{AF}}\left(P_{\mathrm{AF}}^{\text {erg }}\left(\bar{\gamma}, \lambda^{*}\right), \bar{\gamma}\right)-\lambda^{*} P_{\mathrm{AF}}^{\text {erg }}\left(\bar{\gamma}, \lambda^{*}\right)$, where $g\left(\bar{\gamma}, \lambda^{*}\right)$ is given by

$$
g_{\mathrm{AF}}\left(\bar{\gamma}, \lambda^{*}\right)=\lambda^{*}\left\langle P_{\text {supp }}\left(\bar{\gamma}, \lambda^{*}\right)\right\rangle-R_{\text {supp }}\left(\bar{\gamma}, \lambda^{*}\right)
$$

which can be interpreted as the relative cost in allocating the supplemental power.

We now need to establish a few properties of the supplemental cost function which will help us find the optimal $w^{*}(\bar{\gamma})$. Using the expressions for $P_{\mathrm{AF}}^{\mathrm{erg}}\left(\bar{\gamma}, \lambda^{*}\right), P_{\mathrm{AF}}^{r_{0}}(\bar{\gamma})$ 
from (5), (6), one can derive after some algebra that $\left\langle P_{\text {supp }}\left(\bar{\gamma}, \lambda^{*}\right)\right\rangle=\left[e^{2 r_{0}} \frac{\left(\gamma_{3}+c\right)^{2}}{2 \gamma_{3}\left(\gamma_{1}+c\right)^{2}}-\frac{1}{2 \lambda^{*}}\right]^{+}$and $R_{\text {supp }}\left(\bar{\gamma}, \lambda^{*}\right)=\left[r_{0}-\frac{1}{2} \log \left(\frac{\gamma_{3}\left(\gamma_{1}+c\right)^{2}}{\lambda^{*}\left(\gamma_{3}+c\right)^{2}}\right)\right]^{+}$, where $c=$ $\sqrt{\gamma_{1} \gamma_{3}-\gamma_{1} \gamma_{2}+\gamma_{2} \gamma_{3}}$. So clearly, if $P_{\text {supp }}\left(\bar{\gamma}, \lambda^{*}\right)=0$, then $R_{\text {supp }}\left(\bar{\gamma}, \lambda^{*}\right)=0$ and furthermore $g_{\mathrm{AF}}\left(\bar{\gamma}, \lambda^{*}\right)=0$. Otherwise if $P_{\text {supp }}\left(\bar{\gamma}, \lambda^{*}\right)>0, R_{\text {supp }}\left(\bar{\gamma}, \lambda^{*}\right)>0$ and therefore

$$
\begin{aligned}
g_{\mathrm{AF}}\left(\bar{\gamma}, \lambda^{*}\right)=\lambda^{*} & \exp ^{2 r_{0}} \frac{\left(\gamma_{3}+c\right)^{2}}{2 \gamma_{3}\left(\gamma_{1}+c\right)^{2}}-\left(r_{0}+\frac{1}{2}\right) \ldots \\
+ & \frac{1}{2} \log \left(\frac{\gamma_{3}\left(\gamma_{1}+c\right)^{2}}{\lambda^{*}\left(\gamma_{3}+c\right)^{2}}\right) .
\end{aligned}
$$

Denoting $y=\lambda^{*} e^{2 r_{0}} \frac{\left(\gamma_{3}+c\right)^{2}}{2 \gamma_{3}\left(\gamma_{1}+c\right)^{2}}$, it is easy to see that $g_{\mathrm{AF}}\left(\bar{\gamma}, \lambda^{*}\right)=\frac{1}{2}[y-\log y+1]$. If $P_{\text {supp }}\left(\bar{\gamma}, \lambda^{*}\right)>0$, this implies that $y>1$ and in turn, this implies $y>1+\log y$ and therefore $g_{\mathrm{AF}}\left(\bar{\gamma}, \lambda^{*}\right)>0$. Furthermore, one can show that $g_{\mathrm{AF}}\left(\bar{\gamma}, \lambda^{*}\right)$ is a non-increasing function of $\bar{\gamma}$ (by simply computing the derivatives and showing that they are nonpositive).

Using the modified expression for the Lagrangian above and the KKT condition (12e), now it is easy to see that

$$
w^{*}(\bar{\gamma}) \begin{cases}=1 & 0<g_{\mathrm{AF}}\left(\bar{\gamma}, \lambda^{*}\right)<\beta^{*} \\ =v^{*}(\bar{\gamma}) & g_{\mathrm{AF}}\left(\bar{\gamma}, \lambda^{*}\right)=\beta^{*} \\ =0 & g_{\mathrm{AF}}\left(\bar{\gamma}, \lambda^{*}\right)>\beta^{*}\end{cases}
$$

where $0 \leq v^{*}(\bar{\gamma}) \leq 1$ needs to be determined. With the above properties of the supplemental cost function $g_{\mathrm{AF}}\left(\bar{\gamma}, \lambda^{*}\right)$, one can show that $v^{*}(\bar{\gamma})$ can be simply chosen such that $E_{\bar{\gamma}}\left[w^{*}(\bar{\gamma})\right]=1-\epsilon$. See [11] for further details.

Combining all the above results, one can finally state the optimum power allocation solution to Problem (1):

Theorem 1: If Problem (1) is feasible, an optimal power allocation solution for the AF case is given by

$$
P_{\mathrm{AF}}^{*}(\bar{\gamma})=P_{\mathrm{AF}}^{\mathrm{erg}}\left(\bar{\gamma}, \lambda^{*}\right)+X_{w^{*}}(\bar{\gamma}) P_{\text {supp }}\left(\bar{\gamma}, \lambda^{*}\right)
$$

where

$$
w^{*}(\bar{\gamma}) \begin{cases}=1 & 0<g_{\mathrm{AF}}\left(\bar{\gamma}, \lambda^{*}\right)<\beta^{*} \\ =v^{*}(\bar{\gamma}) & g_{\mathrm{AF}}\left(\bar{\gamma}, \lambda^{*}\right)=\beta^{*} \\ =0 & g_{\mathrm{AF}}\left(\bar{\gamma}, \lambda^{*}\right)>\beta^{*}\end{cases}
$$

and $\lambda^{*}, \beta^{*}$ and $0 \leq v^{*}(\bar{\gamma}) \leq 1$ are solutions to $E_{\bar{\gamma}}\left[\left\langle P_{\mathrm{AF}}^{*}(\bar{\gamma})\right\rangle\right]=P_{a v}, E_{\bar{\gamma}}\left[w^{*}(\bar{\gamma})\right]=1-\epsilon$.

Note that the interpretation of the above solution is that when the supplemental power cost is greater than a certain threshold due to the channel being poor, then the supplemental allocation is turned off and power is only allocated to maximize the expected achievable rate, but this is not enough to meet the basic rate and an outage happens. When the supplemental power cost is less than this threshold, then the expected achievable rate maximizing power allocation achieves rate $\geq r_{0}$. At the boundary set $g_{\mathrm{AF}}\left(\bar{\gamma}, \lambda^{*}\right)=\beta^{*}$, a randomization is necessary to determine whether or not to turn on the supplemental power. Note that this randomization is not necessary when $\bar{\gamma}$ is distributed with a continuous probability distribution function resulting in the the boundary set having a measure zero. Finally, all the above derivations can be replicated for the DF case, and the optimum power allocation to Problem (1) in the DF case can be similarly obtained as a switched policy between the expected achievable rate maximizing power allocation and the basic rate power allocation.

\section{NUMERICAL RESUlts}

In this section, we present some simulation studies conducted with typical slow fading channels, where both $\mathrm{AF}$ and DF half-duplex protocols are compared with the direct transmission scheme. It is assumed that none of the links (S-D, S-R, and R-D) has line of sight (LOS) and all links undergo statistically independent Rayleigh fading (albeit with different means). It is assumed that the channel triple $\bar{\gamma}=\left(\gamma_{1}, \gamma_{2}, \gamma_{3}\right)$ are exponentially distributed with means given by the inverse of the node distances with a path loss factor equal to 4 . In order to avoid unnecessary computational complexity, we normalize the S-D distance to 1 , and furthermore, we assume that the relay node is located on the straight line between the source and destination with $d_{\mathrm{S}-\mathrm{R}}=0.4$.

Figure 2 provides a comparison among Ergodic Capacity (EC) (expected achievable rate for the cooperative case), Service Outage (SO) Capacity, and, Outage Capacity (OC). Both SO and OC have the outage constraint at $5 \%$ and the outage threshold at $r_{0}=0.1$ (nats/Hz/Transmission). It is shown that both $\mathrm{AF}$ and $\mathrm{DF}$ perform much better than DT in terms of expected achievable rates, and more importantly, the outage constraint affects the service outage capacity substantially when it comes to the DT scheme: the SO capacity falls down to the outage capacity quite fast when the power limitation turns out to be strict; whereas, those of the two relay protocols are always close to their Ergodic Capacity even in the relatively low SNR region.

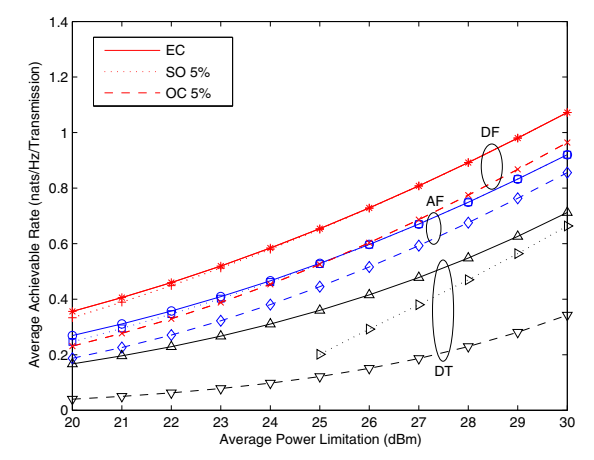

Fig. 2. Ergodic Capacity, Service Outage Capacity, and Outage Capacity

Meanwhile, the corresponding minimum average power, which makes the service outage problem (1) solvable, is given in Figure 3. The graph illustrates that the DT scheme is more power hungry for both $r_{0}=0.5$ and 0.05 (nats/Hz/Transmission). In addition, Figure 4 shows that when the rate threshold $r_{0}$ increases from 0.1 to 1 (in nats/Hz/Transmission), the service outage capacity drops dramatically for the DT scheme, while the two cooperative relay schemes are not affected much. This fact clearly illustrates that the $\mathrm{AF}$ and $\mathrm{DF}$ schemes are more robust to the outage constraints, which is due to the advantage gained by exploiting the relay route for communication. In this simulation, The average power limitation equals to $0 \mathrm{~dB}$ (1 Watt) and the outage probability is $5 \%$.

All the above simulations seem to illustrate that the DF protocol achieves better performance than its counterparts, but it is not always the case. The following graph shows 


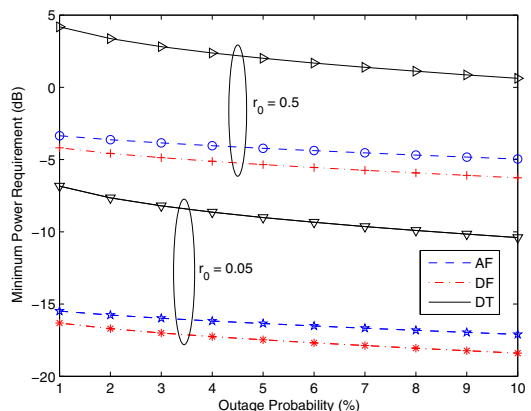

Fig. 3. Minimum Required Powers for AF, DF, and DT

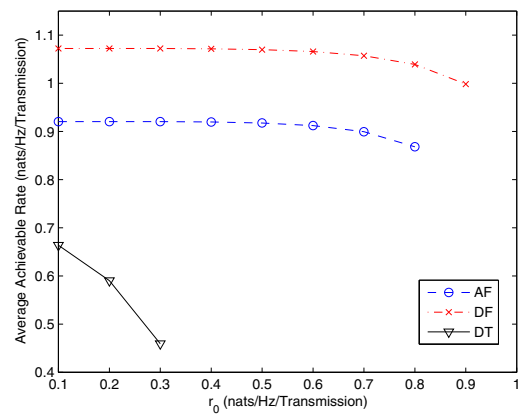

Fig. 4. Service Outage Capacity with Increasing Rate Threshold

the service outage constrained expected achievable rates when the relay node slides along the straight line between the source and destination node. In this situation, the required outage probability is $5 \%$ under the rate threshold $r_{0}=0.01$ (nats/Hz/Transmission), and the average power constraint is $23 \mathrm{dBm}$. At the beginning, the DF scheme dominates over the AF scheme, but its performance declines dramatically when the relay is approaching to the destination. As expected, when the relay is close to the destination, not only does the achievable rate of the AF protocol exceed that of the DF case, but also the DT rate becomes comparable with it, as also shown in [15].

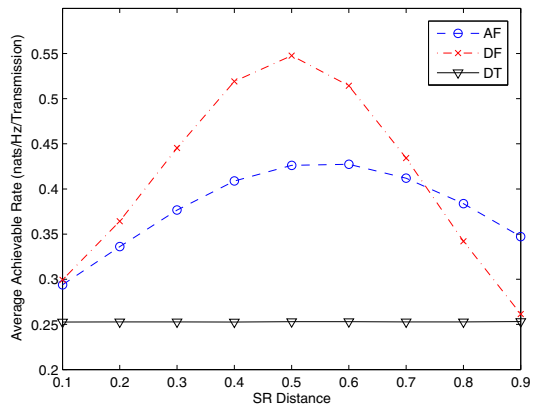

Fig. 5. Service Outage Capacity for different SR distances

\section{CONCLUSIONS}

In this paper we have studied a novel optimum power allocation problem for a three node relay network with slow block fading channels. With full CSI at the transmitters at the source and the relay, this power allocation maximizes the expected achievable rate subject a long term average power constraint and a maximum outage probability constraint. It is shown that this optimum scheme is a switched policy between two deterministic power allocation policies - the expected achievable rate maximizing power allocation and a basic rate power allocation. Numerical results are presented to demonstrate the benefits of cooperation as opposed to direct transmission. Future work will investigate similar problems for relay networks with partial CSI or quantized channel feedback.

\section{REFERENCES}

[1] E. C. van der Meulen, "Three-terminal communication channels," Adv. Appl. Prob., vol. 3, pp. 120-154, 1971.

[2] T. M. Cover and A. E. Gamal, "Capacity theorems for the relay channel," IEEE Trans. on Inform. Theory, vol. IT-25, no. 5, pp. 572584, Sep. 1979.

[3] J. N. Laneman, D. N. C. Tse, and G. W. Wornell, "Cooperative diversity in wireless networks: Efficient protocols and outage behavior," IEEE Transactions on Information Theory, vol. 50, no. 12, pp. 3062 - 3080, Dec. 2004

[4] V. Stankovic, A. Host-Madsen, and Z. Xiong, "Cooperative diversity for wireless ad hoc networks," IEEE Signal Processing Magazine, pp. 37-49, September 2006.

[5] A. J. Goldsmith and P. Varaiya, "Capacity of fading channels with channel side information," IEEE Transactions on Information Theory, vol. 43, pp. 1986-1992, November 1997.

[6] S. V. Hanly and D. N. C. Tse, "Multi-access fading channels - part II: Delay-limited capacities," IEEE Transactions on Information Theory, vol. 44, pp. 2816-2831, November 1998.

[7] G. Caire, G. Taricco, and E. Biglieri, "Optimum power control over fading channels," IEEE Transactions on Information Theory, vol. 45, pp. 1468-1489, July 1999.

[8] A. Host-Madsen and J. Zhang, "Capacity bounds and power allocation for wireless relay channels," IEEE Trans. on Inform. Theory, vol. 51, no. 6, pp. 2020-2040, June 2005.

[9] N. Ahmed, M. A. Khojastepour, and B. Aazhang, "Outage minimization and optimal power control for fading relay channel," in IEEE Inform. Theory Workshop (ITW), San Antonio, TX, USA, Oct. 2004, pp. $458-462$.

[10] J. Luo, L. Lin, R. Yates, and P. Spasojevic, "Service outage based power and rate allocation," IEEE Transactions on Information Theory, vol. 49, no. 1, pp. 323-330, January 2003.

[11] J. Luo, R. Yates, and P. Spasojevic, "Service outage based power and rate allocation for parallel fading channels," IEEE Transactions on Information Theory, vol. 51, no. 7, pp. 2594 - 2611, July 2005.

[12] J. Tang and X. Zhang, "Cross-layer resource allocation over wireless relay networks for quality of service provisioning," IEEE JSAC, vol. 25, no. 4, pp. 645-656, May 2007.

[13] N. Ahmed, M. A. Khojastepour, A. Sabarwal, and B. Aazhang, "Outage minimization with limited feedback for the fading relay channel," IEEE Transactions on Communications, vol. 54, no. 4, April 2006.

[14] S. Boyd and L. Vandenberghe, Convex Optimization. Cambridge University Press, 2004.

[15] J. C. F. Li and S. Dey, "Delay-constrained achievable rate maximization in wireless relay networks with causal feedback," in Proc. of The 9th IEEE International Workshop on Signal Processing Advances in Wireless Communications (SPAWC), July 2008. 